ARTICLE

Received 9 May 2014 | Accepted 5 Nov 2014 | Published 18 Dec $2014 \quad$ DOl: 10.1038/ncomms6760

\title{
Anisotropic softening of magnetic excitations along the nodal direction in superconducting cuprates
}

\author{
M. Guarise1, B. Dalla Piazza1', H. Berger ${ }^{1}$, E. Giannini², T. Schmitt ${ }^{3}$, H.M. Rønnow ${ }^{1,4}$, \\ G.A. Sawatzky ${ }^{5}$, J. van den Brink ${ }^{6}$, D. Altenfeld ${ }^{7}$, I. Eremin ${ }^{7,8} \&$ M. Grioni ${ }^{1}$
}

The high- $T_{\mathrm{c}}$ cuprate superconductors are close to antiferromagnetic order. Recent measurements of magnetic excitations have reported an intriguing similarity to the spin wavesmagnons-of the antiferromagnetic insulating parent compounds, suggesting that magnons may survive in damped, broadened form throughout the phase diagram. Here we show by resonant inelastic $\mathrm{X}$-ray scattering on $\mathrm{Bi}_{2} \mathrm{Sr}_{2} \mathrm{CaCu}_{2} \mathrm{O}_{8+\delta}$ (Bi-2212) that the analogy with spin waves is only partial. The magnon-like features collapse along the nodal direction in momentum space and exhibit a photon energy dependence markedly different from the Mott-insulating case. These observations can be naturally described by the continuum of charge and spin excitations of correlated electrons. The persistence of damped magnons could favour scenarios for superconductivity built from quasiparticles coupled to spin fluctuations. However, excitation spectra composed of particle-hole excitations suggest that superconductivity emerges from a coherent treatment of electronic spin and charge in the form of quasiparticles with very strong magnetic correlations.

\footnotetext{
${ }^{1}$ Institute of Condensed Matter Physics (ICMP), Ecole Polytechnique Fédérale de Lausanne (EPFL), CH-1015 Lausanne, Switzerland. ${ }^{2}$ Département de Physique de la Matière Condensée, Université de Genève, 24 Quai Ernest-Ansermet, 1211 Geneva, Switzerland. ${ }^{3}$ Swiss Light Source, Paul Scherrer Institut, CH-5232 Villigen PSI, Switzerland. ${ }^{4}$ RIKEN Centre for Emergent Matter Science (CEMS), Wako 351-0198, Japan. ${ }^{5}$ Department of Physics \& Astronomy, University of British Columbia, Vancouver, British Columbia, Canada V6T 1Z1. ${ }^{6}$ Institute for Theoretical Solid State Physics, IFW Dresden, D01171 Dresden, Germany. ${ }^{7}$ Institut für Theoretische Physik III, Ruhr-Universität Bochum, D-44801 Bochum, Germany. ${ }^{8}$ National University of Science and Technology 'MISiS', Moscow 119049, Russian Federation. Correspondence and requests for materials should be addressed to M.G. (email: marco.grioni@epfl.ch).
} 
O ne of the most distinctive and surprising features of the cuprate superconductors (SC) is their proximity to an antiferromagnetic (AFM) phase. Understanding what survives of magnetic correlations when the AFM insulator is doped is an outstanding issue. It is especially important for theoretical scenarios where magnetic fluctuations provide the pairing interaction at the origin of high-temperature superconductivity ${ }^{1}$. Theoretical studies of spin dynamics in layered cuprates can be broadly separated into two complementary approaches that work well in opposite limits. The insulating parent compounds are well described in terms of local spins and the Heisenberg hamiltonian ${ }^{2}$. The evolution of spin excitations on introducing a finite amount of carriers is treated by assuming a mixture of local spins described by the superexchange interaction $J$ and itinerant carriers dispersing with hopping integral $t$, such as the $t-J$ model $^{3,4}$. Another class of theories build on an itinerant description, where the system's behaviour is primarily governed by interactions at energies smaller than the fermionic bandwidth, while contributions from higher energies only lead to renormalizations. In this itinerant approach, correlations are usually introduced through the random-phase approximation $(\mathrm{RPA})^{5-7}$, where the spin response is a continuum of electronhole excitations, albeit this approach remains under debate ${ }^{8,9}$. These two approaches can be seen as approximations of the Hubbard model, which probably contains the relevant physics, including superconductivity ${ }^{10}$, and could form the basis of a comprehensive theory that bridges the gap between these two limits. Indeed, numerical calculations on clusters of finite size have shown to be consistent with experimentally measured electronic and magnetic excitation spectra ${ }^{11,12}$. However, a generally valid solution to the Hubbard model remains out of reach. Therefore, new experiments probing both spin and charge dynamics in the crucial intermediate doping range are especially valuable. A key question is whether the excitations of the manybody system can be separated into weakly hybridizing spin and charge excitations, or whether the spin and charge signals are two response functions to the same underlying dynamics.

Neutrons are a standard probe of magnetic excitations, and have provided the full magnon dispersion in selected insulating parent compounds ${ }^{13-15}$. In doped compounds, neutron spectroscopy has provided great insight on the low-energy part of the magnetic excitation spectrum, including an hourglass anomaly, the opening of a spin gap and build-up of a resonance in the superconducting state, which seems to be a generic feature in the cuprates and in some iron-based $\mathrm{SC}^{16-18}$. This anomaly may be at the origin of characteristic features in the electron spectral function measured by angle-resolved photoemission ${ }^{19}$. Dispersive excitations have been reported up to $150 \mathrm{meV}$ (refs 20,21), above which neutron spectroscopy becomes increasingly challenging.

An independent picture of magnetic and charge excitations has now been gained by resonant inelastic X-ray scattering (RIXS) experiments at the $\mathrm{Cu} \mathrm{K}$ edge ${ }^{22}$ and $\mathrm{Cu} \mathrm{L}_{3}$ edge $^{23-28}$ in the cuprates and at the $\mathrm{Fe}_{3}$ edge in the iron pnictides ${ }^{29}$. RIXS offers some interesting advantages, such as the possibility of measuring small-volume samples, additional flexibility from polarization control and complementary information from the $\mathrm{Cu}$ (single magnons) and $\mathrm{O}$ (two magnons) edges ${ }^{30}$. RIXS has enabled a complete mapping of the spin waves in the insulating cuprates. Namely, it revealed dispersive excitations along the magnetic zone boundary, in contrast to the predictions of the simple twodimensional (2D) Heisenberg model, and therefore established the importance of longer-range interactions ${ }^{31}$. A significant breakthrough was the observation by RIXS in several families of SC cuprates of dispersions closely resembling the spin waves of the parent compounds, but progressively broadened with increasing doping ${ }^{32-35}$. In all these cases, the dispersion was studied only along the anti-nodal (100) direction, parallel to the $\mathrm{Cu}-\mathrm{O}$ bonds in the $\mathrm{CuO}_{2}$ planes, for which the $d$-wave superconducting gap is the largest. Here we present highresolution $\mathrm{Cu}$ L-edge RIXS data for optimally doped, underdoped and undoped $\mathrm{Bi}_{2} \mathrm{Sr}_{2} \mathrm{CaCu}_{2} \mathrm{O}_{8+\delta}(\mathrm{Bi}-2212)$ samples, for both the nodal (110) and the anti-nodal (100) directions. We find well-defined dispersive features similar to the magnons of the insulating parent compound along the anti-nodal direction. However, along the nodal direction, these excitations collapse, with spectral weight extending down to zero-energy transfer. While the dispersive anti-nodal excitations could be interpreted as originating from correlated paramagnetic (PM) local moments, we show that both the nodal and the anti-nodal directions are qualitatively described by the continuum of spin excitations calculated for correlated itinerant electrons.

\section{Results}

The doping and momentum dependence of the RIXS spectra. Figure 1 presents RIXS data for $\mathrm{Bi}_{1.6} \mathrm{~Pb}_{0.4} \mathrm{Sr}_{2} \mathrm{YCu}_{2} \mathrm{O}_{8+\delta}$ (BSYCO; insulator), for underdoped $\mathrm{SC} \mathrm{Bi}_{1.6} \mathrm{~Pb}_{0.4} \mathrm{Sr}_{2} \mathrm{Ca}_{0.95} \mathrm{Y}_{0.05} \mathrm{Cu}_{2} \mathrm{O}_{8+\delta}$ (UD-2212; $T_{c}=70 \mathrm{~K}$ ) and for optimally doped $\mathrm{Bi}_{2} \mathrm{Sr}_{2} \mathrm{CaCu}_{2} \mathrm{O}_{8}+\delta$ (OPT-2212; $\left.T_{c}=92 \mathrm{~K}\right)$. All spectra shown in the figure were collected at $20 \mathrm{~K}$ with $\sigma$-polarized light, and the incident photon energy $(930.5 \mathrm{eV})$ coincides with the peak of the $\mathrm{Cu} \mathrm{L}$ $\mathrm{X}$-ray absorption line shape (Fig. 1a). The scattering geometry is illustrated in Fig. 1b. The intensity of the scattered photons is plotted as a function of the energy loss, defined as the difference between the incident and emitted photon energies, for selected values of $q$, the transferred momentum projected on the $a b$ plane. The spectra have been normalized to the same integrated intensity in a window encompassing the manifold of $d d$ excitations around $2 \mathrm{eV}$.

Figure 1c,d shows spectra of insulating BSYCO, measured along the (100) and (110) high-symmetry directions. The line shapes are similar to those of other insulating cuprates, namely, $\mathrm{La}_{2} \mathrm{CuO}_{4}$ $(\mathrm{LCO})^{24}$ and $\mathrm{Sr}_{2} \mathrm{CuO}_{2} \mathrm{Cl}_{2}$ (SCOC) $)^{25}$. One can identify the elastic peak, broadened by non-resolved phonon sidebands, and a sharp magnon peak, dispersing from zero energy at $q=0$ to $\sim 0.3 \mathrm{eV}$ at the boundary of the magnetic Brillouin zone (BZ). The magnon energy is $\sim 60 \mathrm{meV}$ larger at $(\pi, 0)$ than at $(\pi / 2, \pi / 2)$, due to interactions beyond nearest-neighbour spins, as already observed for LCO by neutrons ${ }^{13,14}$ and discussed elsewhere ${ }^{31}$. The high-energy tail of the magnon peak belongs to the multi-magnon continuum.

Data for the UD-2212 sample are illustrated in Fig. 1e,f. A dispersive inelastic feature is visible along the anti-nodal (100) direction (Fig. 1e). It is broader and relatively less intense than in BSYCO, but the dispersion is similar. This is consistent with the results on Bi-2212 in ref. 33. More generally, the momentumdependent RIXS line shape is qualitatively similar to that of other SC cuprates along the anti-nodal direction ${ }^{32}$. The data in Fig. 1g,h for OPT-2212 show that the RIXS spectral function is fairly insensitive to the doping level, as previously reported from experiments performed with both $\sigma$ - and $\pi$-polarized light ${ }^{32-35}$ Along the nodal (110) direction, the line shape is asymmetric and $\sim 4$ times broader than the resolution-limited elastic peak, revealing an underlying inelastic component. At variance with the (100) direction, it is not possible to identify a dispersive feature that could be interpreted as a broadened version of the magnon peak of the insulator. This is reinforced by a detailed analysis of the spectral line shape. Figure 2 illustrates representative spectra of UD-2212, for two values of momentum transfer along the (100) and (110) directions, together with fits performed along the lines in ref. 32. Each spectrum is decomposed into the sum of a paramagnon line 
a

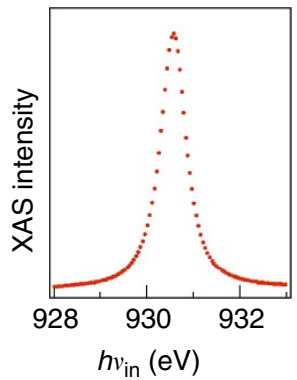

b

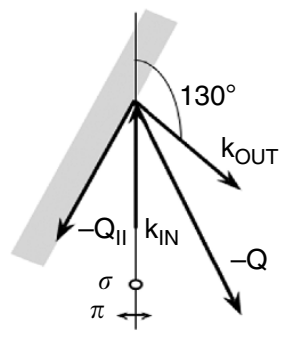

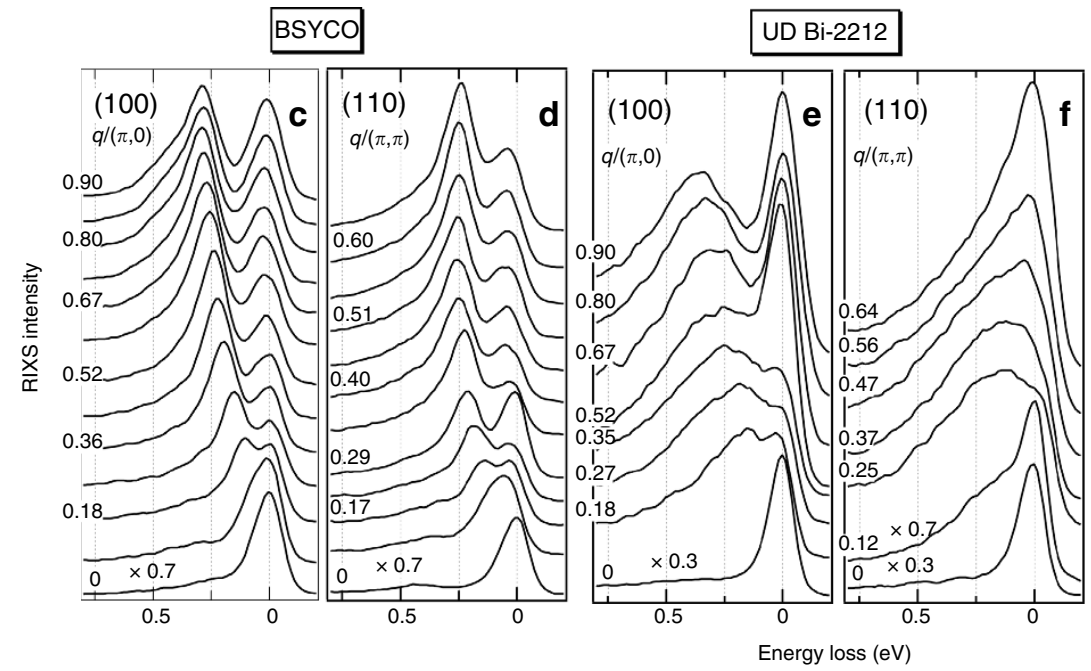

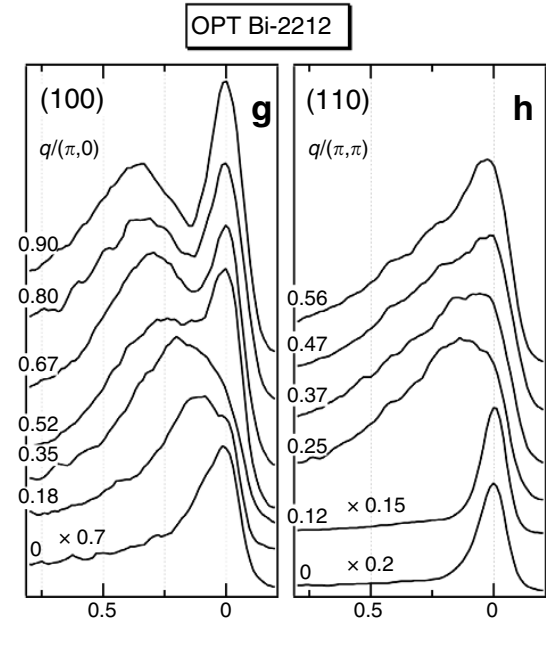

Figure 1 | Overview of the RIXS data. (a) Typical $\mathrm{Cu} \mathrm{L}_{3} \mathrm{X}$-ray absorption (XAS) line shape of an insulating cuprate. The peak of the line shape defines the resonance energy for the RIXS experiment. (b) Schematics of the experimental geometry in the horizontal scattering plane, which contains the sample's (001) direction. (c,d) Cu $L_{3}$ RIXS spectra ( $\sigma$ pol.) of insulating BSYCO, measured at $20 \mathrm{~K}$ along the (100) and (110) high-symmetry directions. The incident energy coincides with the peak of the $L_{3} X A S$. The incidence (emission) angle, measured from the sample normal, varied between $25^{\circ}\left(-25^{\circ}\right)$ at $q=0$ and $85^{\circ}\left(35^{\circ}\right)$ at the largest $q$. Energy loss is the difference between the energies of the incident and the emitted photons. (e,f) UD-2212; $(\mathbf{g}, \mathbf{h})$ OPT-2212.

shape, an elastic peak and a sloping background representing the tail of the higher-lying $d d$ manifold. Satisfactory fits are possible for both spectra along (100). By contrast, a good agreement can never be achieved for the two representative spectra along the nodal direction. Alternatively, the spectra along the (100) direction could be reasonably well described by a suitably broadened magnon peak plus a multi-magnon tail, as in ref. 25, but such an approach would also fail along the nodal direction. Such discrepancy could not be appreciated from the previously published RIXS data. The anisotropic response is illustrated by the energy-momentum intensity map for UD-2212 (Fig. 3) where, for clarity, a resolution-limited elastic peak has been subtracted. The magnon dispersion for BSYCO, superimposed on the map, was extracted from the experimental spectra in Fig. 1 using the same analysis in ref. 25. The RIXS intensity follows the spin-wave dispersion of the insulator along the (100) direction. By contrast, along the (110) direction, the peak of the spectral weight distribution for the superconducting sample is consistently found below the magnon energy.

The evolution of the RIXS spectra with incident photon energy. The data in Figs 1 and 3 suggest that the spin-wave picture built on AFM-correlated local spins, which well describes the insulating cuprates, may not be the most appropriate starting point to describe the metallic (superconducting) phase. A dramatic distinction between the two cases is revealed by the photon energy- dependent RIXS data in Fig. 4. The schematic diagrams of Fig. 4a,b show a RIXS transition from the ground state $\mathbf{G}$ to an excited final state $\mathbf{F}$ in two different situations. In Fig. 4 a, the coherent secondorder optical transition goes through a sharp intermediate level I. This illustrates $\mathrm{Cu} \mathrm{L}_{3}$ RIXS in an insulating cuprate, where the intermediate state $\mathbf{I}$ is the single $2 p^{5} 3 d^{10}$ level and the final state $\mathbf{F}$ is the ground state plus a magnon. In this case, both at and above threshold, the photon energy loss is constant and equal to the energy separation between $\mathbf{G}$ and F. In Fig. 4b, by contrast, the intermediate state is a continuum, as in a metal with a well-defined Fermi surface. Above threshold, this opens the possibility of a real transition, followed by an incoherent radiative de-excitation (fluorescence) from a lower-energy state. The energy difference between the incident and emitted photons-the energy loss-now increases with the incident energy. RIXS measurements as a function of the incident photon energy above threshold can therefore distinguish the two situations.

Figure 4c shows spectra of insulating SCOC and UD-2212 measured near $(\pi, 0)$ at various incident energies at and above the $\mathrm{L}_{3}$ threshold. In SCOC, the magnetic part of the response exhibit an intensity modulation and minor modifications of the line shape as a function of incident energy, but the corresponding energy loss remains constant, as expected. The case of UD-2212 is quite different. Here we observe a linear energy shift of both the $d d$ manifold and the low-energy features (arrows) away from the elastic peak. A few hundred meV's above threshold, the magnon peak is still visible in SCOC, but it has disappeared in the SC. This 


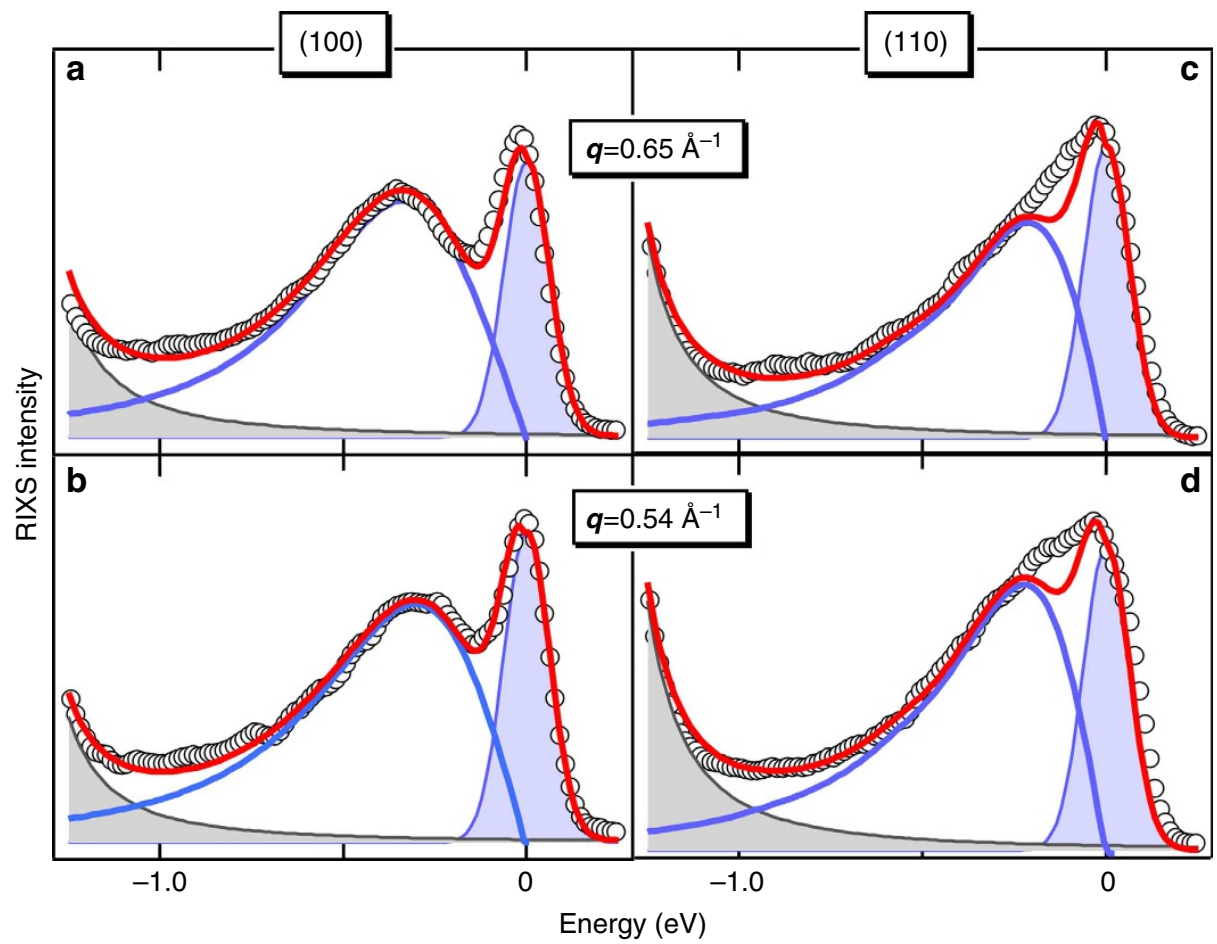

Figure 2 | Line-shape analysis. Spectra of UD-2212 for $q=0.65 \AA^{-1}$ (a) and $q=0.54 \AA^{-1}$ (b) along the (100) direction are analysed with the model in ref. 32. The best fit (red line) is the sum of an elastic peak, a tail from the $d d$ manifold and a paramagnon line shape. The same analysis is repeated in $\mathbf{c}, \mathbf{d}$ for the (110) direction.

clearly demonstrates that, even in the anti-nodal direction, for a description of the low-energy excitations in RIXS it is imperative to consider the continuum of $e-h$ excitations across the Fermi surface.

\section{Discussion}

The previously reported similarity between RIXS spectra in the parent and doped compounds suggests that the doped spectra can be interpreted as damped magnons ${ }^{32-35}$. Calculations of the spin dynamical structure factor $S(\mathbf{q}, \omega)$ performed in the framework of the Hubbard ${ }^{11,12}$ and $t-J$ models ${ }^{32,36}$ have been able to reproduce several aspects of the experimental spectra, namely, the nearly doping-independent dispersion and progressive energy broadening. The results in Fig. 4, on the other hand, indicate that the inelastic spectrum in doped $\mathrm{Bi}-2212$ belongs to the $e-h$ continuum. From a different perspective, it has been argued that the RIXS data could be explained by the band structure alone, without taking into account collective spin fluctuations ${ }^{37}$.

Here we consider the possibility that in the doped compounds the RIXS signal can be described in terms of collective spin excitations from correlated itinerant electrons. This can be simply described in terms of the RPA spin susceptibility ${ }^{38}$, $\chi^{\mathrm{s}}(\mathbf{q}, \omega)=\chi_{0}(\mathbf{q}, \omega) /\left[1-U \chi_{0}(\mathbf{q}, \omega)\right]$, shown in Fig. 5a for the undoped AFM-ordered case and in Fig. 5b for the PM doped case (for further details, see Methods). An interesting feature of this expression is that for collective spin excitations the actual modification of the Lindhard particle-hole response function $\chi_{0}^{\mathrm{s}}(\mathbf{q}, \omega)$ due to the Coulomb interaction $U$ in the RPA denominator occurs only near $(\pi, \pi)$, around the AFM wave vector itself. The dispersive behaviour of $\chi^{\mathrm{s}}$ along the anti-nodal $(0,0) \rightarrow(\pi, 0)$ direction is mainly due to the structure of the Lindhardt function. In Fig. 5b, white circles mark the peak energy in the elastic line-corrected RIXS spectra for UD-2212, extracted from Fig. 3. The calculated RPA $\chi^{\mathrm{s}}$ qualitatively reproduces the salient experimental features, namely: (i) the persistence of a dispersive feature along the $(0,0) \rightarrow(\pi, 0)$ direction; (ii) the

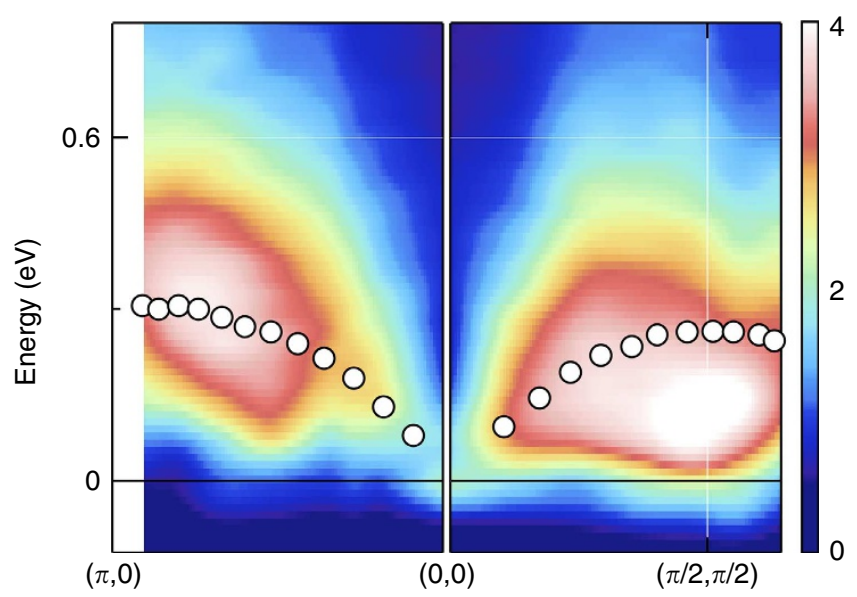

Figure 3 | Comparison of parent and doped compounds. RIXS energy versus momentum intensity map for the UD-2212 sample, after subtraction of a resolution-limited elastic peak. The open symbols track the magnon dispersion of insulating BSYCO, extracted from Fig. 1.

collapse of the excitations to lower energy along the $(0,0) \rightarrow(\pi, \pi)$ direction; and (iii) being a particle-hole continuum, it naturally explains the incident energy dependence reported in Fig. 4. The dispersive features in $\chi^{\mathrm{s}}$ away from $(\pi, \pi)$ are already present in the original $e-h$ response function, but are enhanced by the RPA denominator. For smaller doping values, these excitations become the true spin-wave excitations of the AFM ground state present in the entire BZ. In this regard, the interpretation of the experimental data is close to that in ref. 37 , with the difference that we assume as a basic starting point that RIXS measures the direct spin excitations.

The calculated dispersion of the spin excitations in Fig. $5 b$ shows additional intense features that are seemingly absent in the 
a

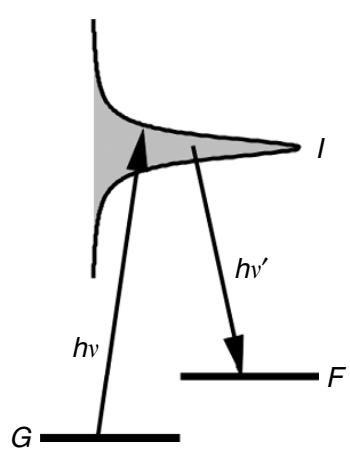

b

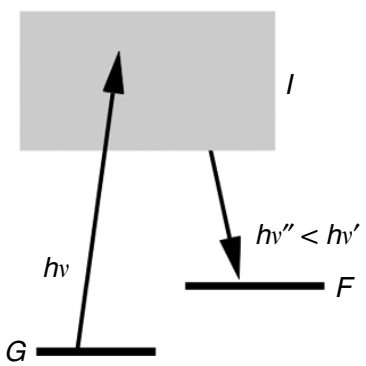

C

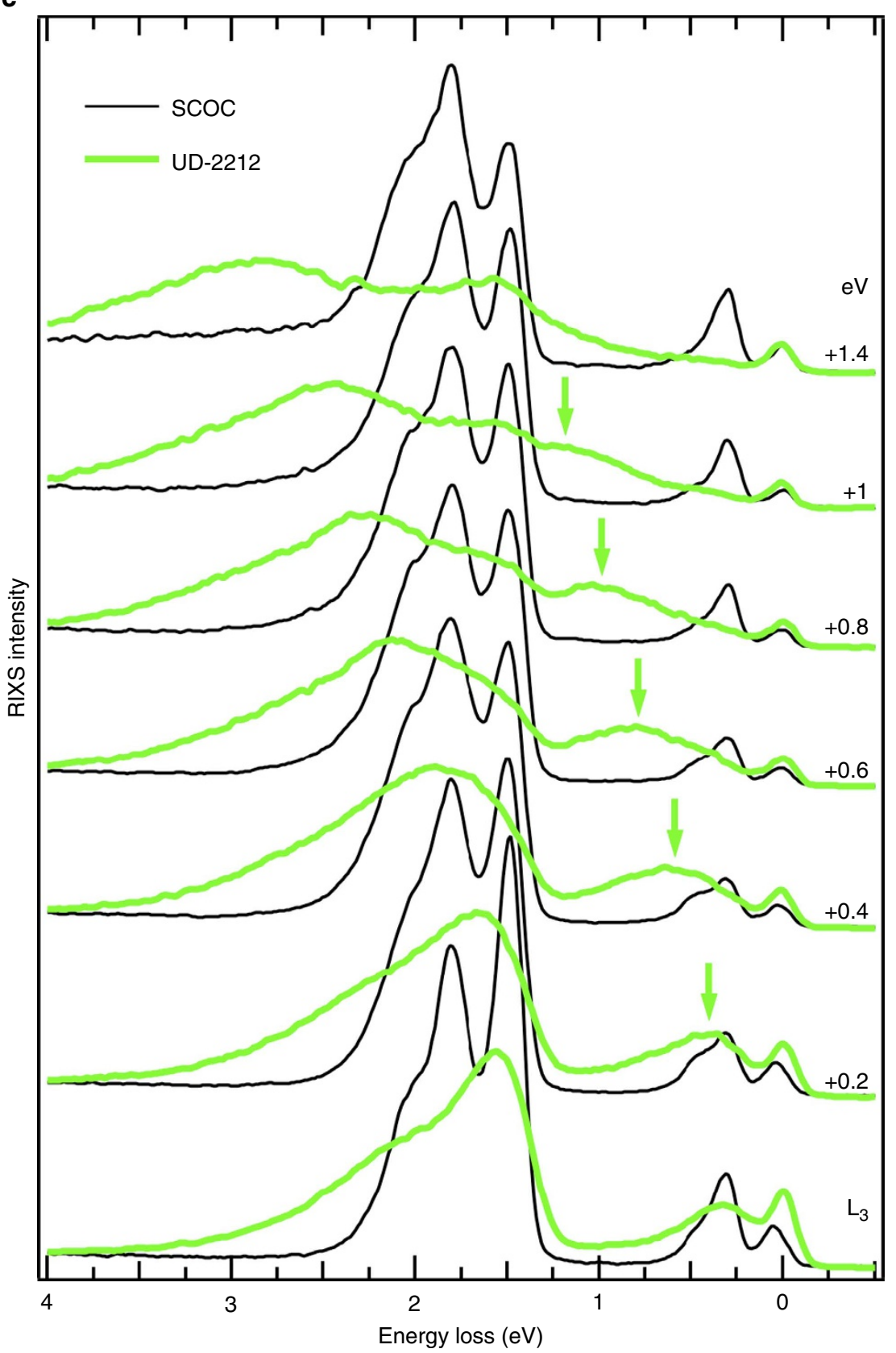

Figure 4 | The evolution of RIXS with incident energy. $(\mathbf{a}, \mathbf{b})$ Schematic total energy diagram for RIXS transitions from the ground state $\mathbf{G}$ to a final excited state $\mathbf{F}$. The intermediate state $\mathbf{I}$ is a single level broadened by the core-hole lifetime in $\mathbf{a}$. A continuum in $\mathbf{b}$ gives rise to fluorescence above threshold. In this case, the measured energy loss is larger than the energy difference between $\mathbf{F}$ and $\mathbf{G}$. (c) RIXS spectra of SCOC and UD-2212 measured up to $1.4 \mathrm{eV}$ above threshold, for $q=0.8(\pi, 0)$. A fluorescence signal (arrows) is observed in UD-2212. 

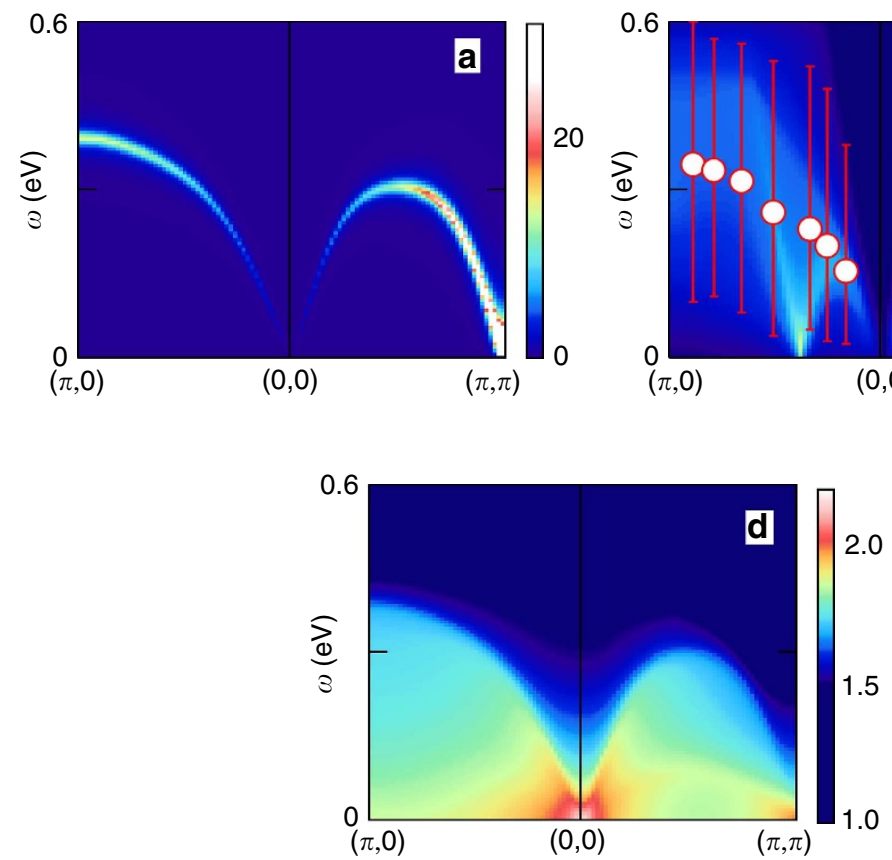

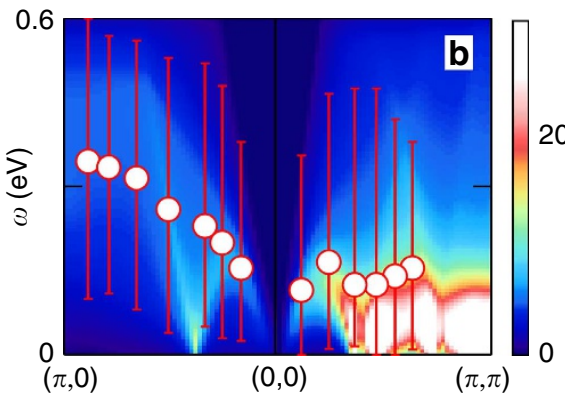

20

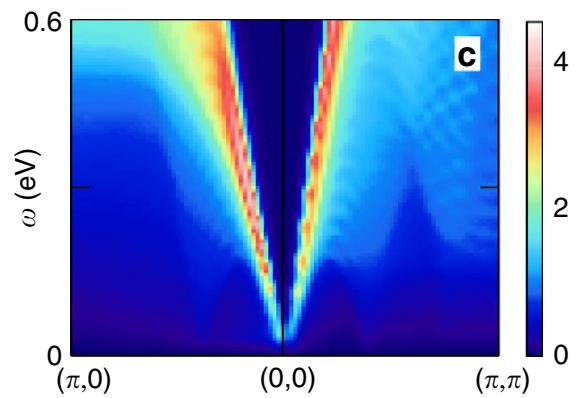

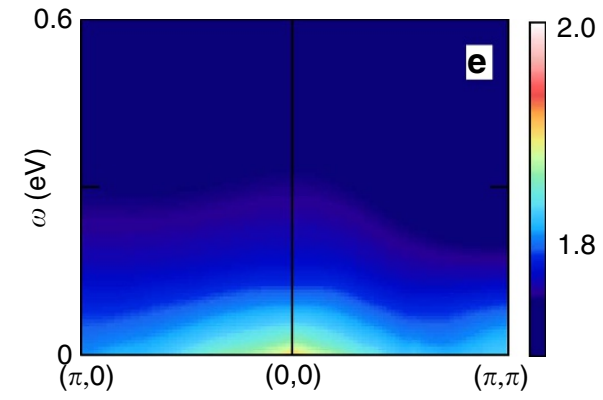

Figure 5 | The calculated RPA susceptibilities. (a) Calculated imaginary part of the RPA magnetic spin susceptibility $\chi^{\mathrm{s}}(\mathbf{q}, \omega)$ for $T=10^{-3} \mathrm{~K}$ in the undoped AFM state (occupancy $n=1)$. (b) $\operatorname{Im}\left[\chi^{\mathrm{s}}(\mathbf{q}, \omega)\right]$ in the paramagnetic state at optimal hole doping $(n=0.85)$. White circles and bars mark the peak energy and width at half maximum of the magnetic response of UD-2212 extracted from the map in Fig. 3. The corresponding charge susceptibility $\operatorname{Im}\left[\chi^{\mathrm{ch}}(\mathbf{q}, \omega)\right]$ is shown in $\mathbf{c}$. For $\mathbf{a}-\mathbf{c}$, we use the following hopping integrals $\left(t_{1}, t_{2}, t_{3}, t_{4}\right)=(126,-36,15,1.5)$ (all in meV) within the tight-binding approximation, which provide a reasonable fit to the band structure of Bi-2212 (ref. 46). The same parameters were also used in ref. 37. (d) The contribution of the collective spin excitations to the charge channel (in arbitrary units) for the case illustrated in a. We used a $500 \times 500 \mathbf{k}$-grid and 200 points for $\omega$ summation. Here, the AFM state was obtained by solving the mean-field gap equations and computing the spin response with respect to the new ground $\operatorname{state}^{38}$. (e) The contribution of collective spin excitations to the charge channel (in arbitrary units) for case $\mathbf{b}$.

experiment. In particular, in the calculations, we find low-energy excitations around $q_{1} \sim(\pi / 3,0)$ and a broad spectral weight distribution below $\omega \sim 0.1 \mathrm{eV}$ between $(\pi / 3, \pi / 3)$ and $(\pi, \pi)$. Within RPA, these excitations arise from regions near the Fermi surface with opposite Fermi velocities, and are usually referred to as $2 k_{\mathrm{F}}$ instabilities. Namely, the $1 \mathrm{D}$ wave vector $q_{1}$ corresponds to the electron-hole scattering between two, nearly parallel, antinodal regions on the Fermi surface near the $(\pi, 0)$ point of the BZ. The second type of scattering originates from the so-called nodal $2 k_{\mathrm{F}}$ instability along the diagonal of the BZ. Due to finite doping, this wave vector is incommensurate and smaller than $(\pi, \pi)$. In principle, these excitations should be visible in RIXS. However, recent calculations ${ }^{37}$, based on the same tight-binding parametrization, show that their intensities are strongly influenced by the RIXS matrix elements. They should become more visible in the strongly overdoped regime where both the proximity to the Mott insulator, which influences the Fermi surface, and the quasiparticle lifetime become less important.

For completeness, we also present in Fig. 5c the chargeresponse function. As mentioned above, within an itinerant approach, the collective charge and spin excitations arise initially from the particle-hole scattering of the electronic states located near the Fermi level. The only difference within the RPA treatment of the single-band Hubbard model is that summing corresponding diagrams yields in the PM-state different denominators for the charge and the PM spin susceptibilities. While the spin response is enhanced within RPA, the charge response is suppressed as $\chi^{\mathrm{ch}}(\mathbf{q}, \omega)=\chi_{0}(\mathbf{q}, \omega) /\left[1+U \chi_{0}(\mathbf{q}, \omega)\right]$. Nevertheless, the gross features of both functions look rather similar, except for the difference in the intensity and the downward shift in energy of the spin excitation. In particular, low-energy features around $q_{1} \sim(\pi / 3,0)$ and $(\pi / 3, \pi / 3)$ in the charge response are very similar to, but weaker than, the corresponding spin excitations. Therefore, in the doped compounds, both spin-flip and non-spin-flip processes contribute often at the same energies, thus making it difficult to disentangle them.

Interestingly, the strong spin fluctuations in doped cuprates also influence the excitations in the charge-response function. It has been argued ${ }^{39}$ that the remarkable correspondence between the position of the spin resonance observed in the inelastic neutron-scattering experiments and the pair-breaking electronic Raman response in the $\mathrm{A}_{1 \mathrm{~g}}$ symmetry could be a results of the collective spin fluctuations, transferred to the charge response via renormalization of the vertex function. Given the similarity between Raman scattering and RIXS, one would expect the same type of diagrams to contribute also to the RIXS intensity. In particular, the spin fluctuation contribution to the charge excitations can be written as ${ }^{39}$ :

$$
I_{\mathrm{ch}}^{\mathrm{sf}}(\mathbf{q}, \omega) \propto \sum_{\mathbf{k}, \Omega} \chi^{\mathrm{s}}(\mathbf{k}, \Omega) \chi^{\mathrm{s}}(\mathbf{k}+\mathbf{q}, \Omega+\omega)
$$

where we set the RIXS matrix elements to unity for the sake of clarity. Then, the total charge response is the one discussed in ref. 37 plus equation (1). We note that collective spin excitations must be well pronounced to be visible in the charge-response function. For example, in Fig. 5d,e) we compare $I_{\mathrm{ch}}^{\mathrm{sf}}$ in the parent AFM phase, where the excitations are the true magnons, and in the itinerant limit. One sees that the structure of the spin excitations is clearly visible only in the case of spin waves, while the itinerant PM response gives a featureless contribution to $I_{\mathrm{ch}}^{\mathrm{sf}}$. This indicates that spin fluctuations strongly influence the charge RIXS response in the undoped and underdoped cuprates but will become less visible in the overdoped cuprates. 
The anisotropy and energy dependence of the RIXS response reported here reveal new aspects of the magnetic excitations in the superconducting cuprates that need to be incorporated in a theory of the electronic structure of these materials. One can anticipate further progress from a more systematic survey of these features across the phase diagram. Specifically, studying how the incident energy dependence evolves from Raman-type response in the insulating parent compound to fluorescence-type response in the superconductor-for example, as a cross-over or as an abrupt transition-could provide a new type of insight to the entanglement of localization and strongly correlated quasiparticles. In addition, experiments implementing polarization analysis of both the incident and scattered beams would be especially valuable, as they would enable unambiguous separation of the magnetic signal from other spectral contributions ${ }^{12}$. Such experiments pose severe technical challenges, but will soon become possible in a new generation of RIXS instruments.

\section{Methods}

Cuprate single-crystal growth. Single crystals of optimally doped

$\mathrm{Bi}_{2} \mathrm{Sr}_{2} \mathrm{CaCu}_{2} \mathrm{O}_{8+\delta}$ were grown by the travelling-solvent floating-zone method in a commercial two-mirror furnace (Cyberstar), under a $93 \% \mathrm{Ar}-7 \% \mathrm{O}_{2}$ atmosphere. The pre-reacted precursor rod (feed) and the crystallization seed were counter rotating at $\approx 20$ r.p.m. and travelled through the hot zone at $0.2 \mathrm{~mm}$ per hour. The growth conditions at the flat liquid-solid interface were kept stable for several days. Plate-like crystals $2-3 \mathrm{~mm}$ long and $0.1-0.2 \mathrm{~mm}$ thick were cleaved (perpendicularly to the $c$ axis) from the as-grown rod and annealed at $500^{\circ} \mathrm{C}$ in $p\left(\mathrm{O}_{2}\right)=10^{-3}$ bar for $\approx 100 \mathrm{~h}$ to fix and homogenize the optimal oxygen content. The critical temperature extracted for the magnetic transition was $92 \mathrm{~K}$, with a transition width narrower than $2 \mathrm{~K}$. Single crystals of insulating BSYCO and SCOC and of SCunderdoped $\mathrm{Bi}_{1.6} \mathrm{~Pb}_{0.4} \mathrm{Sr}_{2} \mathrm{Ca}_{0.95} \mathrm{Y}_{0.05} \mathrm{Cu}_{2} \mathrm{O}_{8+\delta}\left(\mathrm{UD}-2212 ; T_{\mathrm{c}}=70 \mathrm{~K}\right.$ ) were grown using a self-flux method in the form of large platelets perpendicular to the $c$ axis. All samples were characterized by X-ray diffraction and resistivity measurements.

The RIXS experiment. RIXS is a second-order optical process that probes chargeneutral single-particle and collective excitations, with charge or spin character ${ }^{30}$. RIXS measurements at the $\mathrm{Cu} \mathrm{L}_{3}$ absorption edge $\left(2 p_{3 / 2} \rightarrow 3 d\right.$; Fig. 1a) were performed with the SAXES spectrometer ${ }^{40}$ of the ADRESS beam line ${ }^{41}$ at the Swiss Light Source, Paul Scherrer Institut (Villigen, Switzerland). The soft X-ray beam from the undulator source was linearly polarized either within ( $\pi$ polarization) or perpendicular to ( $\sigma$ polarization) the horizontal scattering plane. The scattering geometry is schematically illustrated in Fig. 1b. The fixed scattering angle was $130^{\circ}$, corresponding to a momentum transfer $Q=0.85 \AA^{-1}$. Samples were mounted with the $c$ axis and either the (100) or the (110) direction in the scattering plane. The projection $Q_{\|}=q$ of $\mathbf{Q}$ in the $a b$ plane could be varied between $\pm 0.73 \AA^{-1}$ by rotating the crystal around a vertical axis. The energy calibration and the experimental resolution $\Delta E=130 \mathrm{meV}$ were determined from repeated measurements of the elastic peak of a polycrystalline carbon sample. The incidence (emission) angle, measured from the sample normal, varied between $25^{\circ}\left(-25^{\circ}\right)$ at $q=0$ and $85^{\circ}\left(35^{\circ}\right)$. Self-absorption of the scattered beam is negligible over the whole range of transferred momenta. The magnetic RIXS cross-section exhibits a strong polarization dependence near grazing emission. By contrast, in the nearnormal to grazing incidence geometry adopted here, the cross-sections for $\sigma$ and $\pi$ polarizations are similar ${ }^{42}$. Spectra measured with $\sigma$ polarization exhibit a stronger elastic peak, which provides a useful internal energy calibration.

The RPA calculation. We used an RPA formalism within a single-band Hubbard model,

$$
\mathcal{H}=\sum_{\mathbf{k} \sigma} \varepsilon_{\mathbf{k}} c_{\mathbf{k} \sigma}^{\dagger} c_{\mathbf{k} \sigma}+\sum_{\mathbf{k}, \mathbf{k}^{\prime}, \mathbf{q}, \sigma} U c_{\mathbf{k} \sigma}^{\dagger} c_{\mathbf{k}+\mathbf{q} \sigma} c_{\mathbf{k}^{\prime}+\mathbf{q} \bar{\sigma}}^{\dagger} c_{\mathbf{k}^{\prime} \bar{\sigma}}
$$

to describe the evolution of the spin excitations from the parent AFM state to the SC state at optimal doping. Here, $c_{\mathbf{k} \sigma}^{\dagger}\left(c_{\mathbf{k} \sigma}\right)$ creates (annihilates) an electron with spin $\sigma$ and momentum $\mathbf{k}$ with the normal state tight-binding energy dispersion $\varepsilon_{\mathbf{k}}=-2 t_{1}\left(\cos k_{x}+\cos k_{y}\right)-4 t_{2} \cos k_{x} \cos k_{y}-2 t_{3}\left(\cos 2 k_{x}+\cos 2 k_{y}\right)-2 t_{4}(\cos$ $\left.2 k_{x} \cos k_{y}+\cos k_{x} \cos 2 k_{y}\right)-\mu$ with $\left(t_{1}, t_{2}, t_{3}, t_{4}\right)=(126,-36,15,1.5)$ (all in $\mathrm{meV})$. The chemical potential $\mu$ controls the doping, chemical doping $x$, defined as $n=1+x$

First, we analyze the excitations in the parent AFM state. The Hubbard interaction term in the Hamiltonian equation (S1) leads in the Hartree-Fock factorization to the emergence of the commensurate AFM order, with corresponding spin-wave-type excitations in the transverse spin susceptibility ${ }^{43}$ After decoupling the Hubbard interaction via a mean-field approximation, and diagonalizing the resulting mean-field Hamiltonian via a conventional spin density wave Bogoliubov transformation, one obtains two electronic bands (labelled $\alpha$ and $\beta)$ in the reduced $\mathrm{BZ}$ with dispersions $E_{\mathbf{k}}^{\alpha, \beta}=\varepsilon_{\mathbf{k}}^{+} \pm \sqrt{\left(\varepsilon_{\mathbf{k}}^{-}\right)^{2}+W^{2}}$, where $W=$ $U / 2 \sum_{\mathbf{k}^{\prime}, \sigma}\left\langle c_{\mathbf{k}^{\prime}}^{\dagger}+\mathbf{Q}, \sigma c_{\mathbf{k}^{\prime}, \sigma}\right\rangle \operatorname{sgn} \sigma$ is the spin density wave order parameter that is determined self-consistently for a given $U$, for which we took $3.2 t_{1}$, and $\varepsilon_{\mathbf{k}}^{ \pm}=\left(\varepsilon_{\mathbf{k}} \pm \varepsilon_{\mathbf{k}}+\mathbf{Q}\right) / 2$. For completeness, the self-consistent determination of the chemical potential must also be included.

The dynamical spin susceptibility for the transverse, +- , components is then defined as $\chi^{+-}\left(\mathbf{q}, \mathbf{q}^{\prime}, \Omega\right)=\int \mathrm{d} t\left[\frac{i}{2 N}\left\langle T S_{\mathbf{q}}^{+}(t) S_{-\mathbf{q}}^{-}(0)\right\rangle\right] e^{i \Omega t}$, where we took into account that the AFM ordering at $\mathbf{Q}=(\pi, \pi)$ doubles the unit cell ${ }^{43,44}$. Since commensurate AFM order breaks the translational symmetry and doubles the unit cell, the total susceptibility in the transverse channel is a $2 \times 2$ matrix:

$$
\hat{\chi}^{+-}=\left(\begin{array}{cc}
\chi^{+-}(\mathbf{q}, \mathbf{q}, \Omega) & \chi^{+-}(\mathbf{q}, \mathbf{q}+\mathbf{Q}, \Omega) \\
\chi^{+-}(\mathbf{q}+\mathbf{Q}, \mathbf{q}, \Omega) & \chi^{+-}(\mathbf{q}+\mathbf{Q}, \mathbf{q}+\mathbf{Q}, \Omega)
\end{array}\right) .
$$

Within RPA, the susceptibility is obtained via solving a Dyson equation, and the result can be expressed ${ }^{43}$ as:

$$
\hat{\chi}_{R P A}^{+-}=\left(\hat{1}-\hat{U} \hat{\chi}_{0}^{+-}\right)^{-1} \cdot \hat{\chi}_{0}^{+-},
$$

where $\hat{\chi}_{0}^{+-}$indicates the bare components.

This weak-coupling approach gives an adequate description of the spin excitations in the AFM state of the parent cuprate compounds in quantitative agreement with experiment ${ }^{45}$, as seen in Fig. 5a. An additional advantage of this approach is that it provides a simultaneous information on not only the collective spin excitations of the AFM state, but also the $e-h$ continuum. Furthermore, this approach is naturally extended to the PM state.

At the next stage, we consider hole doping and analyse the spin excitations in the PM state. In this case, the expression for the transverse part of the spin susceptibility is reduced to the standard one $\chi^{\mathrm{s}}(\mathbf{q}, \omega)=\chi_{0}(\mathbf{q}, \omega) /\left[1-U \chi_{0}(\mathbf{q}, \omega)\right]$ where $\chi_{0}(\mathbf{q}, \omega)$ is the Lindhard response function. We further use the value of $U=1.5 t_{1}$ to keep the system in the PM state and not in immediate vicinity to the AFM instability, so that the RPA denominator is far from divergence. Figure $5 \mathrm{~b}$ illustrates the imaginary part of $\chi^{\mathrm{s}}(\mathbf{q}, \omega)$, showing the $e-h$ continuum along the two high-symmetry directions for optimal doping.

\section{References}

1. Chubukov, A. V., Pines, D. \& Schmalian, J. in The Physics of Superconductors Vol. 1 (eds Benneman, K. H. \& Ketterson, J. B.) 495 (Springer, 2003).

2. Hayden, S. M. et al. High-energy spin waves in $\mathrm{La}_{2} \mathrm{CuO}_{4}$. Phys. Rev. Lett. 67, 3622-3625 (2007).

3. Shimahara, H. \& Takada, S. Fragility of the antiferromagnetic long-range-order and spin correlation in the two-dimensional $t-J$ model. J. Phys. Soc. Jpn 61, 989-997 (1992).

4. Sega, I., Prelovsek, P. \& Bonca, J. Magnetic fluctuations and resonant peak in cuprates: towards a microscopic theory. Phys. Rev. B 68, 054524 (2003).

5. Brinckmann, J. \& Lee, P. A. Renormalized mean-Peld theory of neutron scattering in cuprate superconductors. Phys. Rev. B 65, 014502 (2007).

6. Manske, D., Eremin, I. \& Bennemann, K. H. Analysis of the resonance peak and magnetic coherence seen in inelastic neutron scattering of cuprate superconductors: a consistent picture with tunneling and conductivity data. Phys. Rev. B 63, 054517 (2001).

7. Abanov, Ar., Chubukov, A. V., Eschrig, M., Norman, M. R. \& Schmalian, J. Neutron resonance in the cuprates and its effect on fermionic excitations. Phys. Rev. Lett. 89, 177002 (2002).

8. Anderson, P. W. Is there glue in cuprate superconductors? Science 316, 1705-1707 (2007).

9. $\mathrm{Xu}, \mathrm{G}$. et al. Testing the itinerancy of spin dynamics in superconducting $\mathrm{Bi}_{2} \mathrm{Sr}_{2} \mathrm{CaCu}_{2} \mathrm{O}_{8+\delta}$. Nat. Phys. 5, 642-646 (2009).

10. Le Hur, K. \& Rice, T. M. Superconductivity close to the Mott state: from condensed-matter systems to superßuidity in optical lattices. Ann. Phys. 324, 1452-1515 (2009).

11. Macridin, A., Jarrel, M., Maier, T. \& Scalapino, D. J. High-energy kink in the single-particle spectra of the two-dimensional Hubbard model. Phys. Rev. Lett. 99, 237001 (2007).

12. Jia, C. J. et al. Persistent spin excitations in doped antiferromagnets revealed by resonant inelastic light scattering. Nat. Commun. 5, 3314 (2014).

13. Coldea, R. et al. Spin waves and electronic interactions in $\mathrm{La}_{2} \mathrm{CuO}_{4}$. Phys. Rev. Lett. 86, 5377-5380 (2001).

14. Headings, N. S., Hayden, S. M., Coldea, R. \& Perring, T. G. Anomalous highenergy spin excitations in the high- $\mathrm{T}_{\mathrm{c}}$ superconductor-parent antiferromagnet $\mathrm{La}_{2} \mathrm{CuO}_{4}$. Phys. Rev. Lett. 105, 247001 (2010).

15. Plumb, K. W., Savici, A. T., Granroth, G. E., Chou, F. C. \& Kim, Y. J. Highenergy continuum of magnetic excitations in the two-dimensional quantum antiferromagnet $\mathrm{Sr}_{2} \mathrm{CuO}_{2} \mathrm{Cl}_{2}$. Phys. Rev. B 89, 180410(R) (2014).

16. Mook, H. A. et al. Spin fluctuations in $\mathrm{YBa}_{2} \mathrm{Cu}_{3} \mathrm{O}_{6.6}$. Nature 395, 580-582 (1998). 
17. Tranquada, J. M. et al. Quantum magnetic excitations from stripes in copper oxide superconductors. Nature 429, 534-538 (2004).

18. Tsyrulin, N. et al. Magnetic hourglass dispersion and its relation to hightemperature superconductivity in iron-tuned $\mathrm{Fe}_{1+y} \mathrm{Te}_{0.7} \mathrm{Se}_{0.3}$. New J. Phys. 14, 073025 (2012)

19. Dahm, T. et al. Strength of the spin-fluctuation-mediated paiting interaction in a high-temperature superconductor. Nat. Phys. 5, 217-221 (2009).

20. Lipscombe, O. J., Hayden, S. M., Vignolle, B., McMorrow, D. F. \& Perring, T. G. Persistence of high-frequency spin fluctuations in overdoped superconducting $\mathrm{La}_{2-x} \mathrm{Sr}_{x} \mathrm{CuO}_{4}$. $(x=0.22)$. Phys. Rev. Lett. 99, 067002 (2007).

21. Vignolle, B. et al. Two energy scales in the spin excitations of the hightemperature superconductor $\mathrm{La}_{2-x} \mathrm{Sr}_{x} \mathrm{CuO}_{4}$. Nat. Phys. 3, 163-167 (2007).

22. Hill, J. P. et al. Observation of a $500 \mathrm{meV}$ collective mode in $\mathrm{La}_{2 ? x} \mathrm{Sr}_{x} \mathrm{CuO}_{4}$ and $\mathrm{Nd}_{2} \mathrm{CuO}_{4}$ using resonant inelastic X-ray scattering. Phys. Rev. Lett. 100, 097001 (2008).

23. Ament, L. J. P. et al. Theoretical demonstration of how the dispersion of magnetic excitations in cuprate compounds can be determined using resonant inelastic X-ray scattering. Phys. Rev. Lett. 103, 117003 (2009).

24. Braicovich, L. et al. Magnetic excitations and phase separation in the underdoped $\mathrm{La}_{22} \mathrm{Sr}_{x} \mathrm{CuO}_{4}$ superconductor measured by resonant inelastic X-ray scattering. Phys. Rev. Lett. 104, 077002 (2010).

25. Guarise, M. et al. Measurement of magnetic excitations in the two-dimensional antiferromagnetic $\mathrm{Sr}_{2} \mathrm{CuO}_{2} \mathrm{Cl}_{2}$ insulator using resonant $\mathrm{X}$-ray scattering: evidence for extended interactions. Phys. Rev. Lett. 105, 157006 (2010).

26. Haverkort, M. W. et al. Theory of resonant inelastic X-ray scattering by collective magnetic excitations. Phys. Rev. Lett. 105, 167404 (2010).

27. Igarashi, J.-I. \& Nagao, T. Magnetic excitations in L-edge resonant inelastic x-ray scattering from cuprate compounds. Phys. Rev. B 85, 064421 (2012).

28. Ghiringhelli, G. et al. Long-range incommensurate charge fluctuations in $(\mathrm{Y}, \mathrm{Nd}) \mathrm{Ba}_{2} \mathrm{Cu}_{3} \mathrm{O}_{6+\mathrm{x}}$. Science 337, 821-825 (2012).

29. Zhou, K. J. et al. Persistent high-energy spin excitations in iron-pnictide superconductors. Nat. Commun. 4, 1470 (2013).

30. Ament, L. J. P., van Veenendaal, M., Devereaux, T. P., Hill, J. P. \& van den Brink, J. Resonant inelastic x-ray scattering studies of elementary excitations. Rev. Mod. Phys. 83, 705-767 (2011).

31. Dalla Piazza, B. et al. Unified one-band Hubbard model for magnetic and electronic spectra of the parent compounds of cuprate superconductors. Phys. Rev. B 85, 100508(R) (2012)

32. Le Tacon, M. et al. Intense paramagnon excitations in a large family of hightemperature superconductors. Nat. Phys. 7, 725-730 (2011).

33. Dean, M. P. M. et al. High-energy magnetic excitations in the cuprate superconductor $\mathrm{Bi}_{2} \mathrm{Sr}_{2} \mathrm{CaCu}_{2} \mathrm{O}_{8}+\delta$ : towards a unified description of its electronic and magnetic degrees of freedom. Phys. Rev. Lett. 110, 147001 (2013).

34. Le Tacon, M. et al. Dispersive spin excitations in highly overdoped cuprates revealed by resonant inelastic X-ray scattering. Phys. Rev. B 88, 020501(R) (2013).

35. Dean, M. P. M. et al. Persistence of magnetic excitations in $\mathrm{La}_{2-x} \mathrm{Sr}_{x} \mathrm{CuO}_{4}$ from the undoped insulator to the heavily overdoped non-superconducting metal. Nat. Mater. 12, 1019-1023 (2013).

36. Chen, W. \& Sushkov, O. P. Implications of resonant inelastic x-ray scattering data for theoretical models of cuprates. Phys. Rev. B 88, 184501 (2013).

37. Benjamin, D., Klich, I. \& Demler, E. Single-band model of resonant inelastic $\mathrm{X}$-ray scattering by quasiparticles in high- $\mathrm{T}_{\mathrm{c}}$ cuprates. Phys. Rev. Lett. 112, 247002 (2014).
38. Rowe, W., Knolle, J., Eremin, I. \& Hischfeld, P. J. Spin excitations in layered antiferromagnetic metals and superconductors. Phys. Rev. B 86, 134513 (2012).

39. Venturini, F., Michelucci, U., Devereaux, T. P. \& Kampf, A. P. Collective spin fluctuation mode and Raman scattering in superconducting cuprates. Phys. Rev. B 62, 15204-15207 (2000)

40. Ghiringhelli, G. et al. SAXES, a high resolution spectrometer for resonant x-ray emission in the 400-1600 eV energy range. Rev. Sci. Instrum. 77, 113108 (2006)

41. Strocov, V. N. et al. High-resolution soft X-ray beamline ADRESS at the Swiss Light Source for resonant inelastic X-ray scattering and angle-resolved photoelectron spectroscopies. J. Synchrotron Rad. 17, 631-643 (2010).

42. Braicovich, L. et al. Momentum and polarization dependence of single-magnon spectral weight for $\mathrm{Cu} \mathrm{L}_{3}$-edge resonant inelastic X-ray scattering from layered cuprates. Phys. Rev. B 81, 174533 (2010).

43. Schrieffer, J. R., Wen, X. G. \& Zhang, S. C. Dynamic spin fluctuations and the bag mechanism of high- $\mathrm{T}_{c}$ superconductivity. Phys. Rev. B 39, 11663-11679 (1989).

44. Chubukov, A. V. \& Frenkel, D. M. Renormalized perturbation theory of magnetic instabilities in the two-dimensional Hubbard model at small doping. Phys. Rev. B 46, 11884-11901 (1992).

45. Peres, N. M. R. \& Araujo, M. A. N. Spin-wave dispersion in $\mathrm{La}_{2} \mathrm{CuO}_{4}$. Phys. Rev. B 65, 132404 (2002).

46. Markiewicz, R. S., Sahrakorpi, S., Lindroos, M., Lin, H. \& Bansil, A. One-band tight-binding model parametrization of the high- $\mathrm{T}_{c}$ cuprates including the effect of $\mathrm{k}_{z}$ dispersion. Phys. Rev. B 72, 054519 (2005).

\section{Acknowledgements}

The work at Lausanne is supported by the Swiss NSF. The work of I.E. is partially supported by the Ministry of Education and Science of the Russian Federation in the framework of the Program to Increase Competitiveness of NUST 'MISiS' (No. K2-2014015). We acknowledge stimulating discussions with E. Demler and D. Benjamin and thank R. Gaal for the resistivity measurements. The SAXES instrument was jointly built by the Paul Scherrer Institut (Villigen, Switzerland), Politecnico di Milano (Italy) and École Polytechnique Fédérale de Lausanne (EPFL, Switzerland)

\section{Author contributions}

M.Gu. and B.D.P. performed RIXS measurements with the support of T.S.; H.B. and E.G. grew the single-crystal samples; M.Gu., H.M.R. and M.G. analysed the data and discussed the results with G.A.S., J.v.d.B. and I.E.; D.A. and I.E. performed the calculations; and M.G., I.E. and H.M.R. wrote the paper.

\section{Additional information}

Competing financial interests: The authors declare no competing financial interests.

Reprints and permission information is available online at http://npg.nature.com/ reprintsandpermissions/

How to cite this article: Guarise, M. et al. Anisotropic softening of magnetic excitations along the nodal direction in superconducting cuprates. Nat. Commun. 5:5760 doi: $10.1038 /$ ncomms6760 (2014). 